\section{Recomendações do Departamento de Neuroendocrinologia da Sociedade Brasileira de Endocrinologia e Metabologia para o Diagnóstico e Tratamento da Acromegalia no Brasil}

\author{
Recommendations of Neuroendocrinology Department \\ from Brazilian Society of Endocrinology and Metabolism \\ for Diagnosis and Treatment of Acromegaly in Brazil
}

Leonardo Vieira Neto',2, Julio Abucham ${ }^{3}$, Luiz Antônio de Araujo4, Cesar L. Boguszewski ${ }^{5}$, Marcello D. Bronstein ${ }^{6}$, Mauro Czepielewski ${ }^{7}$, Raquel S. Jallad' ${ }^{6}$, Nina R. de C. Musolino ${ }^{8}$, Luciana A. Naves', Antônio Ribeiro-Oliveira Júnior $^{10}$, Lucio Vilar ${ }^{1}$, Manuel dos S. Faria' ${ }^{12}$, Mônica R. Gadelha ${ }^{1,13}$

U m dos objetivos do Departamento de Neuroendocrinologia da Sociedade Brasileira de Endocrinologia com a publicação (1) foi tornar claro quais pacientes devem ser submetidos ao rastreamento para acromegalia e como confirmar laboratorialmente seu diagnóstico. As dosagens recomendadas são a do GH e IGF-I, as quais devem ser feitas em pacientes com suspeita clínica de acromegalia (1-3). Não existem, no entanto, diretrizes estabelecendo de forma específica quantos sintomas/sinais clínicos devem estar presentes para levar o paciente a ser submetido ao rastreamento para acromegalia. $\mathrm{O}$ autor da carta enumera os sinais e sintomas inespecíficos presentes na tabela l (1), os quais podem estar presentes na população geral normal ou em outras patologias. Naqueles pacientes com características clínicas típicas da doença, como o embrutecimento facial e o aumento de extremidades, o diagnóstico clínico da acromegalia é, em geral, facilmente estabelecido e confirmado pelo valor alto de IGF-I e o exame de imagem, independente do valor do $\mathrm{GH}$ basal ou no teste oral de tolerância à glicose (TOTG). Há relatos na literatura de pacientes com acromegalia que apresentam supressão do $\mathrm{GH}$ para valores $<0,4 \mu \mathrm{g} / \mathrm{L}$, valor utilizado atualmente como ponte de corte para os ensaios atuais (4). Concordamos com o autor da carta que neste contexto talvez o TOTG seja dispensável. Por outro lado, um valor normal de IGF-I quando há suspeita clínica torna muito improvável o diagnóstico e, nessas situações de dúvida, a dosagem de GH durante o TOTG pode ser útil. Embora o autor enfatize seu trabalho sobre níveis máximos basais de $\mathrm{GH}$ em indivíduos normais pela manhã, não há dados suficientes na literatura que corroborem um nível de corte de $\mathrm{GH}$ acima do qual se estabeleceria com certeza o diagnóstico de acromegalia.

Quanto ao "diagnóstico precoce", é discutível se em uma doença de baixa prevalência é custo-efetivo o rastreamento em pessoas assintomáticas ou com sintomas inespecíficos, devendo-se ter cuidado com a premissa que "obrigatoriamente maior número de indivíduos deverão ter a doença suspeitada e serem investigados laboratorialmente", conforme exposto pelo autor da carta. O objetivo das recomendações não foi de preconizar o rastreamento indiscriminado (o que reduziria o valor preditivo
1 Serviço de Endocrinologia,

Hospital Universitário Clementino Fraga Filho, Universidade Federal do Rio de Janeiro (HUCFF-UFRJ), Rio de Janeiro, RJ, Brasil

2 Serviço de Endocrinologia, Hospital da Lagoa, Rio de Janeiro, RJ, Brasil

${ }^{3}$ Unidade de Neuroendócrino, Escola Paulista de Medicina,

Universidade Federal de São Paulo (Unifesp/EPM), São Paulo, SP, Brasil

${ }^{4}$ Instituto de Endocrinologia de Joinville (IEJE/CAD),

Joinville, SC, Brasil

${ }^{5}$ Serviço de Endocrinologia e

Metabologia, Hospital de Clínicas, Universidade Federal do Paraná (UFPR), Curitiba, PR, Brasil

${ }^{6}$ Serviço de Endocrinologia, Hospital das Clínicas, Faculdade de Medicina da Universidade de São Paulo (FMUSP),

São Paulo, SP, Brasil

7 Serviço de Endocrinologia,

Hospital de Clínicas de Porto Alegre (HCPA), PPG Endocrinologia, Faculdade de Medicina, Universidade Federal do Rio Grande do Sul (UFRGS),

Porto Alegre, RS, Brasil

${ }^{8}$ Divisão de Neurocirurgia Funcional, Instituto de Psiquiatria, Hospital das Clínicas, FMUSP,

São Paulo, SP, Brasil

${ }^{9}$ Serviço de Endocrinologia,

Hospital Universitário de Brasília, Universidade de Brasília

(UnB), Brasília, DF, Brasil

${ }^{10}$ Serviço de Endocrinologia,

Hospital das Clínicas, Universidade Federal de Minas Gerais (UFMG),

Belo Horizonte, MG, Brasil

${ }^{11}$ Serviço de Endocrinologia, Hospital das Clínicas, Universidade Federal de Pernambuco (UFPE), Recife, PE, Brasil

12 Serviço de Endocrinologia, Hospital Universitário Presidente Dutra, Universidade Federal do Maranhão (UFMA), São Luís, MA, Brasil

${ }^{13}$ Serviço de Endocrinologia, Instituto Estadual de Diabetes e Endocrinologia Luiz Capriglione (IEDE), Rio de Janeiro, RJ, Brasil

Correspondência para: Mônica R. Gadelha Rua Prof. Rodolpho Paulo Rocco, 255, 9o andar, Ilha do Fundão 21941-913 - Rio de Janeiro, RJ, Brasil

Recebido em 9/Abr/2011 Aceito em 12/6/2011 
positivo dos testes), mas dentro de um contexto clínico apropriado (1). Nos casos em que as manifestações clínicas são menos específicas e nas situações de suspeita de acromegalia subclínica, como em portadores de incidentalomas hipofisários e adenomas mistos produtores de PRL e GH, as recomendações foram delineadas com base nos dados da literatura e na experiência dos autores, neste caso, todos representantes dos principais centros de referência do Brasil para acromegalia. Concordamos com o autor que, nesse contexto, descartando-se as conhecidas condições clínicas que podem reduzir falsamente o IGF-I, uma dosagem normal afastaria o diagnóstico de acromegalia, porém, pela escassez de dados na literatura, julgamos prudente seguir as mesmas recomendações publicadas previamente por outras sociedades internacionais $(2,5-7)$. Com relação ao $\mathrm{GH}$, saliente-se mais uma vez que não há dados suficientes na literatura para estabelecer valores de corte para GH basal (randômico) acima dos quais seriam considerados "indubitavelmente elevados". Além disso, conforme enfatizado no artigo (1), a abordagem da acromegalia deve ser preferencialmente realizada em centros de referência, locais onde o endocrinologista tem mais condições de interpretar os valores de GH e IGF-I, bem como as possibilidades eventuais de resultados falso-positivos ou falso-negativos.
Declaração: os autores declaram não haver conflitos de interesse científico neste estudo.

\section{REFERÊNCIAS}

1. Vieira Neto L, Abucham J, Araujo LA, Boguszewski CL, Bronstein MD, Czepielewski M, et al. [Recommendations of Neuroendocrinology Department from Brazilian Society of Endocrinology and Metabolism for diagnosis and treatment of acromegaly in Brazil.]. Arq Bras Endocrinol Metabol. 2011;55(2):91-105.

2. Giustina A, Barkan A, Casanueva FF, Cavagnini F, Frohman L, Ho $\mathrm{K}$, et al. Criteria for cure of acromegaly: a consensus statement. $J$ Clin Endocrinol Metab. 2000;85(2):526-9.

3. Boguszewski CL. O laboratório no diagnóstico e seguimento da acromegalia. Arq Bras Endocrinol Metab. 2002;46(1):34-44.

4. Dimaraki EV, Jaffe CA, DeMott-Friberg R, Chandler WF, Barkan AL. Acromegaly with apparently normal GH secretion: implications for diagnosis and follow-up. J Clin Endocrinol Metab. 2002;87(8):3537-42.

5. Giustina A, Chanson P, Bronstein MD, Klibanski A, Lamberts $S$, Casanueva FF, et al. A Consensus on Criteria for Cure of Acromegaly. J Clin Endocrinol Metab. 2010;95(7):3141-8.

6. Barkan A, Bronstein MD, Bruno OD, Cob A, Espinosa-de-los-Monteros AL, Gadelha MR, et al. Management of acromegaly in Latin America: expert panel recommendations. Pituitary. 2010;13(2):168-75.

7. Melmed S, Colao A, Barkan A, Molitch M, Grossman AB, Kleinberg $D$, et al. Guidelines for acromegaly management: an update. J Clin Endocrinol Metab. 2009;94(5):1509-17. 